\title{
Expression Profiling and Bioinformatic Analyses of a Novel Cold Stress-Regulated and Chloroplast-Targeted Protein from Triticum aestivum and Aegilops tauschii
}

\author{
E. Valiellahi (Corresponding author) \\ Institute of Biotechnology, University of Shiraz, Shiraz, Iran \\ E-mail: e_valiellahi@yahoo.com \\ A. Niazi \\ Institute of Biotechnology, University of Shiraz, Shiraz, Iran \\ E-mail: Niazi47@yahoo.com
}

M. Farsi

Department of Biotechnology and Plant Breeding, College of Agriculture

Ferdowsi University of Mashhad, Mashhad, Iran

E-mail: Mohfarsi@yahoo.com

\begin{abstract}
Cold acclimation is a multigenic trait that allows hardy plants to develop efficient tolerance mechanisms needed for winter survival. To determine the genetic nature of these mechanisms, several cold-responsive genes of unknown functions were identified from cold-acclimated wheat (Triticum aestivum). To identify the putative functions and structural features of these new genes, integrated genomic approaches of data mining, expression profiling and bioinformatic predictions were used. We herein report the structural heterogeneity of cDNAs, distribution, low temperature-specificity and protein structure of the identified Wcorl4 gene. Analyses of the cDNA and genomic DNA sequences by Vector NTI 9.0 software, suggested that, Wcorl4 and its related sequences constitute a small multigene family with different intron sizes. The deduced WCOR14 polypeptide, a hydrophobic polypeptide with 140 amino acids $(\mathrm{MW}=13.5 \mathrm{kDa}$ ), showed a high homology to the previously identified wheat and barley COR proteins. No homologous sequences were found in other organisms suggesting that this family is unique to the plant kingdom. The highly homologous signal peptides of WCOR14, BCOR14b and WCS19 contained one putative 14-3-3 protein recognition motif. In this motif, S-residue was predicted as a phosphorylation site and besides this, four other putative phosphorylation sites in WCOR14 were predicted by the NetPhos version 2.0 software. Comparative analyses of gene expression profiling shows that the expression of this gene is correlated with freezing tolerance in cereals.
\end{abstract}

Keywords: Triticum aestivum, Abiotic stress, Cold-acclimation, Expression profile, Wcor 14

\section{Introduction}

To achieve the complete life cycle and reproduction in temperate regions, hardy plants like winter wheat (Triticum aestivum) have developed two major evolutionary adaptive mechanisms: vernalization and cold acclimation (CA). Cold acclimation is triggered by the exposure of plants to low temperature, for certain period of time. During this process, plants exhibit dramatic alterations in their gene expression profiles, which are characterized by the induction of a battery of cold-responsive (Cor) genes (Guy et al. 1985; Guy 1990). Importantly, this adaptive process is believed to be tightly associated with the development of cold/freezing tolerance (Thomashow 1998, 1999). Overwintering plants sense the upcoming winter and delay flowering by postponing the transition from the vegetative to the cold-sensitive reproductive phase (Simpson et al. 1999). In addition, they develop a high degree of freezing tolerance (FT) needed for winter survival (Fowler et al. 1999). Following low temperature (LT) acclimation, some winter cereals can tolerate temperatures as low as $-33^{\circ} \mathrm{C}$. The regulatory mechanisms underlying these two processes and how they are interconnected are far from being fully understood. To gain further knowledge on the strategies that plants use for winter survival, the identification of 
cold-regulated (Cor) genes are needed. A survey of the literature reveals that the expressions of a large number of genes are altered during the process of CA (Thomashow 1999; Breton et al. 2000; Seki et al. 2002).

The genes responsible for CA could be classified into four groups, based on the presumed function of the encoded proteins. The first group comprises genes encoding structural proteins that may be involved in protecting the cells during LT stress. The second group represents those genes that regulate gene expression and signal transduction pathways, such as transcription factors, protein kinases, phosphatases and the enzymes involved in phosphoinoside metabolism. The third group represents genes encoding enzymes involved in the biosynthesis of different osmoprotectants, membrane lipids and those of the antioxidative response. The fourth group contains cold-induced genes encoding proteins of unknown function. To gain insight into the function of these novel proteins, a combination of expression profiling and bioinformatic tools, can be used to predict properties and features that may be important for their function.

Previous studies have shown that, compared with winter varieties, the less hardy spring wheat varieties cannot maintain the expression of Cor genes (e.g. the $W c s 19$ family) at a high level and this differential expression is closely associated with their low degree of FT (Sarhan et al. 1997). As a subsequent step, each novel LT-regulated protein sequence can be analyzed using available bioinformatic tools. These tools help in the identification of sorting signals, conserved posttranslational modifications, transmembrane helices, secondary and tertiary structures. The most recent prediction softwares incorporate machine-learning algorithms in the form of a neural network and a hidden Markov model (Blom et al. 1999; Krogh et al. 2001).

In wheat, one unique member of $C o r$ genes designated as $W c s 19$ encodes a leaf specific and basic $(\mathrm{pI}=8.8)$ protein WCS19 which is also transported into the stromal compartment of the chloroplasts during cold acclimation (Chauvin et al. 1993; Gray et al. 1997). As WCS19 shows a low level of homology with the barley COR14b, we attempted to isolate the orthologue of corl $14 b$. We herein report the structural heterogeneity of cDNAs, distribution, low temperature-specificity and protein structure of the identified Wcor 14 gene. To identify the putative functions and structural features of this new gene, integrated genomic approaches of data mining, expression profiling, and bioinformatic predictions were used. Bread wheat (Triticum aestivum), originated by hybridization of cultivated allotetraploid emmer wheat (T. turgidum ssp. dicoccum, $2 n=4 x=28$, genomes $A A B B$ ) with diploid Aegilops tauschii or Ae. squarrosa $(2 n=2 x=14$, genome DD; Caldwell et al. 2004). Aegilops squarrosa $(\mathrm{n}=7)$ is the donor of the third or $\mathrm{D}$ genome to common wheat and also the donor of the pivotal genome to five polyploid species of the genus Aegilops. This diploid species grows as a predominantly autogamous wild grass or weed in the Middle East. Ae. squarrosa as well as Triticum monococcum and Ae. speltoides constitute a large unexplored gene pool for wheat breeding (Zohary et al. 1969).

\section{Materials and Methods}

\subsection{Plant materials}

Three cultivars of Iranian common wheat (T. aestivum L.), winter-type Azare2, Sardari, Alamoot and wild diploid Ae. tauschii were used. Azare2 has been reported as one of the hardiest cultivars among tetraploid and hexaploid common wheat tested for cold tolerance. Seeds from each of wheat cultivars and Ae. tauschii were planted as separate groups in the same pots $(20 \mathrm{~cm} \times 12 \mathrm{~cm}$ in width and $12 \mathrm{~cm}$ in depth) with soil, and incubated in a growth chamber under the following standard temperature and light conditions; $24 \pm 0.5^{\circ} \mathrm{C}$ with a $14 \mathrm{~h}$ photoperiod at a light intensity of $100-110 \mu \mathrm{m}$ photons $\mathrm{m}^{-2} \mathrm{~s}^{-1}$ provided by cool white fluorescent lamps. Twenty three days-old seedlings were cold-acclimated at $4 \pm 0.5^{\circ} \mathrm{C}$ for different periods under the standard light condition at intensity of $100-110 \mu \mathrm{m}$ photons $\mathrm{m}^{-2} \mathrm{~s}^{-1}$ provided by cool white fluorescent lamps with a 14 hour photoperiod.

\subsection{Genomic PCR analysis}

Total genomic DNA, extracted from leaves of two weeks-old seedlings, were harvested and used for PCR amplification of the genomic Wcor14 sequence. A nested-PCR carried out, where the first amplification was performed using a forward primer (WcorF1:5'-CTCGTCCCACACCGTCAGC-3') and a reverse primer (WcorR1: 5'-TCATTTGCTCACATCCTCGACCGC-3') and the second amplification using a primer (WcorF2: 5'-CTGCCTGCAAACCCCTCCTA-3') and (WcorR2: 5'-CCTCCTCCGTCGCCTGCTTCGCCT-3').

\subsection{Total RNA extraction and cDNA synthesis}

Total RNA was isolated from $100 \mathrm{mg}$ of leaves with RNX-Plus ${ }^{\mathrm{TM}}$ kit according to the manufacturer's specifications. The concentration of total RNA was determined with NanoDrop by measuring absorbance at a wavelength of $260 \mathrm{~nm}$ (A260) and purity was assessed by the ratio of the absorbance values at 260 and $280 \mathrm{~nm}$. For cDNA synthesis one $\mu \mathrm{g}$ total RNA and $0.5 \mu \mathrm{g}$ oligo-dT primer was added and incubated for $5 \mathrm{~min}$ at $70^{\circ} \mathrm{C}$ and then cooled on ice. dNTP $(1 \mathrm{mM})$, Tris buffer $(10 \mathrm{mM})$ and RNAse inhibitor $(40 \mathrm{u} / 20 \mu \mathrm{l})$ was added and for 
$5 \mathrm{~min}$ incubated at $37^{\circ} \mathrm{C}$. Finally, $200 \mathrm{u}$ M-MuLV enzyme was added to cocktail and incubated for $60 \mathrm{~min}$ at $37^{\circ} \mathrm{C}$. Then incubated for $10 \mathrm{~min}$ at $70^{\circ} \mathrm{C}$ in order to inactivate the enzyme. Synthesized cDNA was stored at $-20^{\circ} \mathrm{C}$ (Sambrook and Russell 2001).

\subsection{Semi-quantitative RT-PCR}

Relative semi-quantitative PCR was performed to study the gene expression of $W$ cor 14 .Each PCR reaction was performed in a total volume of $25 \mu \mathrm{l}$ containing, Tris buffer $(10 \mathrm{mM}, \mathrm{pH}=8), \mathrm{MgCl}_{2}(2 \mathrm{mM}), \mathrm{dNTP}$ mix $(0.8$ mM, each dATP, dTTP, dCTP, dGTP $0.2 \mathrm{mM})$, Taq DNA polymerase $(1 \mathrm{u})$, cDNA $(2 \mu \mathrm{l})$, sense primer; 5'-CTGCCTGCAAACCCCTCCTA -3' and antisense primer 5'-CCTCCTCCGTCGCCTGCTTCGCCT-3'(each $0.5 \mu \mathrm{M})$. The product size of $215 \mathrm{bp}$ was expected. To avoid false positives from pseudogenes of contaminating genomic DNA, primer sequences were designed to span intron regions. The PCR condition were as follows, initial denaturation step of $5 \mathrm{~min}$ at $94^{\circ} \mathrm{C}$ was followed by 30 cycles, $45 \mathrm{sec}$ at $94^{\circ} \mathrm{C}, 45 \mathrm{sec}$ at $68^{\circ} \mathrm{C}, 2.3 \mathrm{~min}$ at $72^{\circ} \mathrm{C}$, and end step $5 \mathrm{~min}$ at $72^{\circ} \mathrm{C}$. $\beta$ _tubulin, house keeping gene was used as the reference gene for $W$ cor 14 (Fig.1). The following primer set was designed to isolate a complete open-reading-frame (ORF) from cDNA, product size 423 bp (Fig.2): WcorF: 5'-ATGGCTTCTTCTTCCGTGCTGCT-3' and WcorR: 5'-TCATTTGCTCACATCCTCGACCGC-3'.

\subsection{Gel Electrophoresis}

The semi-quantitative PCR products were loaded onto ethidium bromide stained $1 \%$ agarose gels in TBE buffer. Documentation of agarose gel was done and quantification of amplified products was performed by Total lab software. The intensities of the Wcor 14 mRNA bands were normalized relative to that of $\beta$-tubulin bands by dividing the former by the $\beta$-tubulin specific PCR product densities. $\beta$-tubulin acted as a referenc for sample to sample variation in reverse transcription and PCR conditions, and the extent of degradation and recovery of RNA.

\subsection{Protein isolation and analysis}

Protein extract were prepared from four days cold-acclimated plant by homogenizing $500 \mathrm{mg}$ of leaves in $1 \mathrm{ml}$ of extract buffer (Tris-HCl $0.05 \mathrm{M}, \mathrm{pH} 8,0.02 \% \mathrm{SDS}, 30.3 \%$ urea, and 1\% 2-mercaptoethanol) was added to each micro tube, and centrifuged at $13000 \mathrm{rpm}$ for $10 \mathrm{~min}$ at $4^{\circ} \mathrm{C}$. The supernatant contained dissolved extracted protein ready for experiment purposes. The protein concentration was determined by NanoDrop and 100ng protein was used for experiment. SDS-PAGE gel preparation, running and staining were carried out as standard procedures.

\subsection{Structural Analyses}

For detection of specific targeting sequences, we used PSORT, iPSORT (Nakai and Kanehisa 1992; Bannai et al. 2002; http://psort.nibb.ac.jp), and TargetP v1.01 (Emanuelsson et al. 2000; http://www.cbs.dtu.dk). For TMD prediction, TMHMM (http://www.cbs.dtu.dk) was used (Krogh et al. 2001). Phosphorylation site predicted by NetPhos version 2.0 software (http://genome.cbs.dtu.dk/services/NetPhos). Predictions of secondary structure were carried out by SOPMA available in www.expasy.org (Jaakola et al. 2001). We used I-TASSER server for protein structure prediction (Zhang 2008).

\section{Results and Discussion}

\subsection{Genomic structure of Wcor14}

To further analyze genomic structure of the Wcor14 loci, nested genomic PCR amplification was carried out using primer sets of WcorF1-WcorR1 and WcorF2-WcorR2. In this study, total DNA extracted from Ae. tauschii and three cultivars of Iranian common wheat seedlings, were used as a template. The result showed that there was one sized intron (97 bp intron) delimited by GT-AG in Ae. tauschii Wcor14 coding region and there were two different sized introns (174 bp of intron and $97 \mathrm{bp}$ of intron) delimited by GT-AG in the three cultivars of Iranian common wheat $W$ cor 14 coding region (Fig.3).

\subsection{Structural analysis of Wcor14 cDNAs and their deduced WCOR14 polypeptides}

A period of cold acclimation is an important factor for Wcor14 transcript accumulation. A previous study on the protein profile in response to low temperature in wheat demonstrated that two groups of translatable mRNAs were expressed during cold acclimation (Danyluk et al. 1991). The first group consisted of 18 mRNAs that reached their highest levels of induction after one day of low temperature exposure, but thereafter decreased to undetectable levels. The second group consisted of 53 mRNAs that were also induced rapidly, but maintained their high levels of expression all along four weeks of the experiment period. Among the second group, at least 34 were expressed at higher levels in freezing tolerant winter wheat cultivars than in less tolerant spring wheat 
cultivars and Wcorl4 is in the second group. The identical sequences have been submitted to the GenBank databases (designated as Ae. tauschii Wcor14, accession number FJ670451, T. aestivum cultivar Adle cross cold-responsive protein WCOR14, accession number FJ605270 and T. aestivum cultivar Adle cross cold-responsive protein WCOR14, accession number FJ655857) had 423 bp containing an open reading frame which was predicted to encode a $13.5 \mathrm{kDa}$ acidic $(\mathrm{pI}=4.71)$ polypeptide of 140 amino acid residues. A search in the NCBI database resulted four sequences of T. aestivum encoding Wcor14: Wcor14a (accession number AF207545), Wcor14b (accession number AF207546), WCOR14a (Accession number AF491838) and WCOR14c (AF491837). The deduced amino acid sequence of Ae. tauschii Wcor14 protein (WCOR14) showed 70\% identity with the barley COR14b $(\mathrm{pI}=4.5)$ and $100 \%$ identity with WCOR14a $(\mathrm{pI}=4.71)$ (Fig. 4).

Notably, a stretch of the N-terminal 51 amino acid residues of WCOR14 was nearly identical to that of the barley COR14b (98\% identity with only one amino acid difference), and highly homologous ( $78 \%$ identity) to that of the wheat WCS19. The downstream part of WCOR 14 showed $54 \%$ identity with COR $14 \mathrm{~b}$, but only $34 \%$ identity with WCS19. In contrast to Arabidopsis COR15a, WCOR14 was considerably hydrophobic (59\% hydrophobic residues) similar to COR14b and WCS19 (56\% and 55\%, respectively). The barley COR14b immunologically cross-reacts with a related, chloroplast-imported protein COR14a (Crosatti et al. 1999). $\mathrm{N}$-terminal microsequencing of COR14b was unsuccessful, but that of the N-terminal 11 amino acids of COR14a purified from the chloroplast fraction suggested that it was encoded by a gene cor $14 a$ independent from cor $14 b$. The homology of this partial N-terminal sequence is high with the corresponding part of WCS19, the barley cor14a was suggested to be orthologous to the wheat Wcs 19. Similar to the barley COR14b, however, the N-terminal 51 amino acids of WCOR14a lacked the loosely defined consensus cleavage sequence of (Val/Lle)-X-(Ala/Cys)-Ala which is characteristic for transit peptides that target nuclear-encoded proteins to the stromal compartment of chloroplasts (Gavel and von Heijne 1990). Except for this discrepancy, the sequence had several features in common with the reported chloroplast transit peptides. First, it had a relatively high serine plus threonine content $(12 \%)$, but had no acidic residues. Second, it had an uncharged N-terminal domain (residues 1 to 23 ), a central domain (24 to 41 ) containing 3 positively charged residues and lacking acidic residues (Garnier et al. 1978).

\subsection{COR14 Proteins Contain Conserved Putative Phosphorylation Sites}

Motif searches using the PROSITE, Pfam and Smart databases, did not detect any known motifs. However, the neural network-based NetPhos phosphorylation site prediction software generated several interesting findings (Blom et al. 1999). NDong et al. (2002), reported that the highly homologous signal peptides of WCOR14 and WCS19 contained one putative 14-3-3 protein recognition motif. This amino acid motif was conserved in WCOR15. In this motif, S-residue was predicted as a phosphorylation site by the NetPhos version 2.0 software (http://genome.cbs.dtu.dk/services/NetPhos). Besides this, there were four other putative phosphorylation sites in WCOR14 (Fig. 5). Generally, the 14-3-3 recognition motifs are phosphorylated and continuously interact with the 14-3-3 proteins (May and Soll 2000). The binding of the 14-3-3 proteins to the signal peptides is necessary for the chloroplast precursor proteins to be efficiently transported into chloroplasts (May and Soll 2000). Since both of the WCOR14 and WCOR15 proteins contain a putative 14-3-3 recognition motif in the chloroplast-targeting signal and WCOR15 at least is targeted into chloroplasts in transgenic tobacco plants (Takumi et al. 2003), these phosphorylated proteins might interact with the 14-3-3 proteins to be efficiently transported into chloroplasts of both monocotyledonous and dicotyledonous plants. Analysis using the TMHMM2.0 program (server for prediction of transmembrane helices in proteins) revealed that there is no trnsmembrane helices present in WCOR14 sequences.

\subsection{Gene Expression Studies}

A reverse transcriptase-PCR experiment was carried out to compare the mRNA profile of plants grown at $24^{\circ} \mathrm{C}$ with those of plants exposed at $4^{\circ} \mathrm{C}$. The previous studies showed higher freezing tolerance of winter-hardy common wheat (T. aestivum L.) cv. Mironovska 808 compared with that of a spring-type common wheat cv. Chinese Spring by the simple one-point assay (Kume et al. 2005). It has been demonstrated that such cultivar difference in freezing tolerance could be partly caused by the differential accumulation levels of COR/LEA transcripts during cold acclimation (Vágújfalvi et al. 2000; Kobayashi et al. 2004). We also studied Wcor14 transcript accumulation under the low temperature condition and compared the expression profiles of three Iranian cultivars Wcor 14 genes, and found that they were also induced early and at high levels in Azar2 and Sardari. Transcripts of Wcor 14 rapidly accumulated within 3-6 hours after cold acclimation at $4^{\circ} \mathrm{C}$. The expression patterns clearly showed rapid response to LT in Azar2 and Sardari than Alamoot. There was a sharp increase in $W$ cor 14 transcripts in the leaves of all three genotypes after four days of LT acclimation (Fig. 6). In the non-acclimated control plants, no Wcor14 transcripts were detected. 
To examine the effect of long-term acclimation on Wcor14 transcript accumulation, 23-day-old seedlings were placed under the LT condition and kept for 35days. The amount of Wcor 14 transcript showed a gradual decrease under the long-term acclimation condition The amount of the major transcript reached a maximum at day 4 and thereafter leveled down under the long-term acclimation condition (Fig. 6). The electrophoretic profiles of soluble proteins in three genotypes (Azar2, Alamoot and Sardari) showed the contrasting cold tolerance nature (Fig. 7). Comparison of profiles of proteins extracted from four day acclimated plants indicated that polypeptides of about $14 \mathrm{kDa}$ were the major proteins that accumulated during LT condition. This approximately 14-kDa band was present at a very higher intensity in the acclimated plants than that of control plants (Fig. 7). Immunoblot analysis of the Wcor14 proteins, using polyclonal antibodies showed that accumulation of Wcorl4 protein increased during cold acclimation condition (Kume et al. 2005).

\subsection{Protein structure prediction}

Knowledge of a protein structure provides insight into how it can interact with other proteins, DNA/RNA, and small molecules. It is these interactions which define the protein's function and biological role in an organism. Protein prediction is exacerbated by large amounts of sequence data from whole genome projects and experimentally determining protein structures remains expensive and time consuming. The predicted secondary structure of T.aestivum cultivar Adle cross WCOR14 protein consisted of $68.57 \% \alpha$ helix, $25 \%$ random coil, $5 \%$ extended strand and $1.43 \% \beta$ turns and Ae. tauschii WCOR14 protein consisted of $65 \% \alpha$ helix, $25.71 \%$ random coil, $7.86 \%$ extended strand and $1.43 \% \beta$ turns. The random coil and alpha helix constituted domain comprised the main part of the secondary structure in these proteins. We used I-TASSER server for protein structure prediction (Fig. 8). I-TASSER is a hierarchical protein structure modeling approach based on the secondary-structure enhanced Profile-Profile threading Alignment (PPA) and the iterative implementation of the Threading ASSEmbly Refinement (TASSER) program (Zhang 2008).

Our results strongly suggest that cold acclimation induced protein accumulation plays a crucial role in freezing tolerance of these genotypes. However, subsequent studies have suggested that LT tolerance is affected by other interacting regulatory circuitries (Chinnusamy et al. 2007), besides low temperature, genetic potential, and the factors determining developmental stage, such as vernalization and photoperiod (Mahfoozi et al. 2001). Regulatory loci at the Vrn-A1/Fr1 loci on chromosome 5A have been shown to control expression of Wcor14 (Vágújfalvi et al. 2000; Kobayashi et al. 2005), more specifically regions associated with cbf-lik sequences (Crosatti et al. 2003; Kobayashi et al. 2005). A cis acting element, named C-repeat (CRT) or LT responsive element (LTRE), containing A/GCCGAC motif that forms the core of the DRE sequence, have been shown to regulate LT inducible promoters in Arabidopsis (Baker et al. 1994; Stockinger et al. 1997), Barascica (Jiang et al. 1996), rice (Rabbani et al. 2003) and wheat (Takumi et al. 2003). The transcription factors that interact with the CRT/DRE element are the C-repeat Binding Factor/DRE Binding protein 1 (CBF/DREB1), which first was found in a yeast one-hybrid screen by Stockinger et al (1997). Furthermore, Kume et al. (2005) demonstrated that a cbf homologue, Wcbf2, when activated by LT led to induction and increase in accumulation of Cor/Lea genes such as Wcor14 and Wcor15. Thus the discrepancy in expression between Azare2, Sardari and Alamoot could be due to the influence of the Vrn-A1 locus.

Based on the present results, we conclude that Ae. tauschii Wcor14 is an orthologue of the barley cor14b. The expression of Wcorl4 is low temperature specific. Further, its turnover appeared to be rapid and the expression remains at a high steady-state level during the cold acclimation period. This finding suggests that a major regulatory switch governs the expression of low temperature-responsive genes in wheat. The identification of this genetic system will certainly lead to a better understanding of how low temperature regulates this complex multigenic trait. Efforts can now be focused on the molecular characterization of this system and on the elucidation of the interaction occurring between this regulator and the promoter regions of low temperature-responsive genes. These genes and their products are good candidate for functional analysis using bioinformatics, biochemical, and genetic approaches. Future work should focus on studying the expression of additional cold acclimation genes and accumulation of proteins to get a better understanding of the complex gene interactions that regulate cold acclimation in the field. The level of protein accumulation should be studied to provide a better understanding of how protein accumulation relates to the level of cold tolerance.

\section{Acknowledgments}

We are grateful to Dr. E. Ebrahimie for his helpful comments. This work was supported by grants from the University of Shiraz. 


\section{References}

Baker, S.S., Wilhelm, K.S., \& Thomashow, M.F. (1994). The 5-region of Arabidopsis thaliana cor15a has cis-acting elements that confer cold-, drought- and ABA-regulated gene expression. Plant Mol. Biol., 24, 701-713.

Bannai, H., Tamada, Y., Maruyama, O., Nakai, K., \& Miyano, S. (2002). Extensive feature detection of N-terminal protein sorting signals. Bioinformatics, 18, 298-305.

Blom, N., Gammeltoft, S., \& Brunak, S. (1999). Sequence and structure-based prediction of eukaryotic protein phosphorylation sites. J. Mol. Biol., 294, 1351-1362.

Breton, G., Danyluk, J., Ouellet, F., \& Sarhan F. (2000). Biotechnological applications of plant freezing associated proteins. Biotechnol. Annu. Rev., 6, 59-101.

Caldwell, K.S., Dvorak, J., Lagudah, E.S., Akhunov, E., Luo, M.C., Wolters, P., \& Powell, W. (2004). Sequence polymorphism in polyploid wheat and their d-genome diploid ancestor. Genetics, 167(2), 941-7.

Chauvin, L.P., Houde, M., \& Sarhan, F. (1993). A leaf-specific gene stimulated by light during wheat acclimation to low temperature. Plant. Mol. Biol., 23, 255-265.

Chinnusamy, V., Zhu, J., \& Zhu, J.K. (2007). Cold stress regulation of gene expression in plants. Trends. Plant. Sci., 12, 444-451.

Crosatti, C., Mare, C., Mazzucotelli, E., Selioni, S., Barilli, S., Bassi, R., Dubcovsky, J., Galiba, G., Stanca, A.M., \& Cattivelli, L. (2003). Genetic analysis of the expression of the cold-regulated gene cor14b: a way toward the identification of components of the cold response signal transduction in Triticeae. Can. J. Bot., 81, $1162-1167$.

Danyluk, J., Houde, M., Rassart, E., \& Sarhan, F. (1994). Differential expression of a gene encoding an acidic dehydrin in chilling sensitive and freezing tolerant Gramineae species. FEBS Lett, 344, $20-24$.

Emanuelsson, O., Nielsen, H., Brunak, S., \& von Heijne, G. (2000). Predicting subcellular localization of proteins based on their N-terminal amino acid sequence. J. Mol. Biol., 300, 1005-1016.

Fowler, D.B., Limin, A.E., \& Ritchie, J.T. (1999). Low-temperature tolerance in cereals: model and genetic interpretation. Crop. Sci., 39, 626-633.

Garnier, J., Osguthorpe, D.J., \& Robson, B. (1978). Analysis of the accuracy and implications of simple methods for predicting the secondary structure of globular proteins. J. Mol. Biol., 120, 97-120.

Gray, G.R., Chauvin, L.P., Sarhan, F., \& Huner, N.P.A. (1997). Cold acclimation and freezing tolerance. Plant. Physiol., 114, 467-474.

Guy, C.L. (1990). Cold acclimation and freezing stress tolerance: role of protein metabolism. Annu. Rev. Plant Physiol. Plant Mol. Biol., 41, 187-223.

Guy, C.L., Niemi, K.J., \& Brambl, R. (1985). Altered gene expression during cold acclimation of spinach. Proc. Natl. Acad. Sci,. U.S.A. 82, 3673-3677.

Jaakola, L., Pirtila, A.M., Halonen, M., \& Hohtola, A. (2001). Isolation of high quality RNA from bilberry (Vaccinium myrtillus L.) fruit. Mol Biotechnol., 19,203-210

Jiang, C., Iu, B., \& Singh, J. (1996). Requirement of a CCGAC cis-acting element for cold induction of the BN115 gene from winter Brassica napus. Plant Mol Biol., 30, 679-684.

Kobayashi, F., Takumi, S., Nakata, M., Ohno. R., Nakamura, T., \& Nakamura, C. (2004). Comparative study of the expression profiles of the Cor/Lea gene family in two wheat cultivars with contrasting levels of freezing tolerance. Physiol. Plant, 120, 585-594.

Krogh, A., Larsson, B., von Heijne, G., \& Sonnhammer, E.L. (2001). Predicting transmembrane protein topology with a hidden Markov model: application to complete genomes. J. Mol. Biol., 305, 567-580.

Kume, S., Kobayashi, F., Ishibashi, M., Ohno, R., Nakamura, C., \& Takumi, S. (2005). Differential and coordinated expression of $\mathrm{Cbf}$ and $\mathrm{Cor} / \mathrm{Lea}$ genes during long-term cold acclimation in two wheat cultivars showing distinct levels of freezing tolerance. Genes. Genet. Syst., 80, 185-197.

Mahfoozi, S., Limin, A.E., \& Fowler, D.B. (2001). Influence of vernalization and photoperiod responses on cold hardiness in winter cereals. Crop. Sci., 41, 1006-1011. 
May, T., \& Soll, J. (2000). 14-3-3 proteins form a guidance complex with chloroplast precursor proteins in plants. Plant. Cell, 12, 53-63.

Nakai, K., \& Kanehisa, M. (1992). A knowledge base for predicting protein localization sites in eukaryotic cells. Genomics, 14, 897-911.

NDong, C., Danyluk, J., Wilson, K.E., Pocock, T., Huner, N.P.A., \& Sarhan, F. (2002). Cold-regulated cereal chloroplast late embryogenesis abundanlike proteins. Molecular characterization and functional analysis. Plant. Physiol, 129, 1368-1381.

Rabbani, M.A., Maruyama, K., Abe, H., Khan, M.A., Katsura, K., Ito, Y., Yoshiwara, K., Seki, M., Shinozaki, K., \& Yamaguchi-Shinozaki, K. (2003). Monitoring expression profiles of rice genes under cold, drought, and high-salinity stresses and abscisic acid application using cDNA microarray and RNA gel-blot analyses. Plant. Physiol, 133, 1755-1767.

Sambrook, J., \& Russell, D.W. (2001). Molecular cloning: a laboratory manual. $3^{\text {rd }}$ edition. New York: Cold Spring Harbo, pp. 886-896.

Sarhan, F., Ouellet, F., \& Vazquez-Tello, A. (1997). The wheat wcs120 gene family: a useful model to understand the molecular genetics of freezing tolerance in cereals. Physiol. Plant, 101, 439-445.

Seki, M., Narusaka, M., Ishida, J., Nanjo, T., Fujita, M., Oono, Y., Kamiya, A., Nakajima, M., Enju, A., \& Sakurai, T. (2002). Monitoring the expression profiles of 7000 Arabidopsis genes under drought, cold and high-salinity stresses using a full-length cDNA microarray. Plant. J., 31, 279-292.

Simpson, G.G., Gendall, A.R., \& Dean, C. (1999). When to switch to flowering. Annu. Rev. Cel.l Dev. Biol., 15, 519-550.

Stockinger, E.J., Gilmour, S.J., \& Thomashow, M.F. (1997). Arabidopsis thaliana CBF1 encodes an AP2 domain-containing transcriptional activator that binds to the C-repeat/DRE, a cis-acting DNA regulatory element that stimulates transcription in response to low temperature and water deficit. Proc. Natl. Acad. Sci., USA. 94, 1035-1040.

Takumi, S., Koike, A., Nakata, M., Kume, S., Ohno, R., \& Nakamura, C. (2003). Cold-specific and light-stimulated expression of a wheat (Triticum aestivum L.) Cor gene Wcor15 encoding a chloroplast-targeted protein. J. Exp. Bot., 54, 2265-2274.

Thomashow, M.F. (1998). Role of cold-responsive genes in plant freezing tolerance. Plant Physiol., 118, 1-8.

Thomashow, M.F. (1999). Plant cold acclimation: Freezing tolerance genes and regulatory mechanisms. Annu. Rev. Plant. Physiol. Plant. Mol. Biol., 50, 571-599.

Vágújfalvi, A., Crosatti, C., Galiba, G., Dubcovsky, J., \& Cattivelli, L. (2000). Two loci on wheat chromosome $5 \mathrm{~A}$ regulate the differential cold-dependent expression of the cor $14 \mathrm{~b}$ gene in frost-tolerant and frost-sensitive genotypes. Mol. Genet. Genomics, 263, 194-200.

Zhang, Y. (2008). I-TASSER server for protein 3D structure prediction. BMC Bioinformatics, 9,40

Zohary, D., Harlan, J.R., \& Vardi, A. (1969). The wild diploid progenitors of wheat and their breeding value. Euphytica, 18, 58-65. 


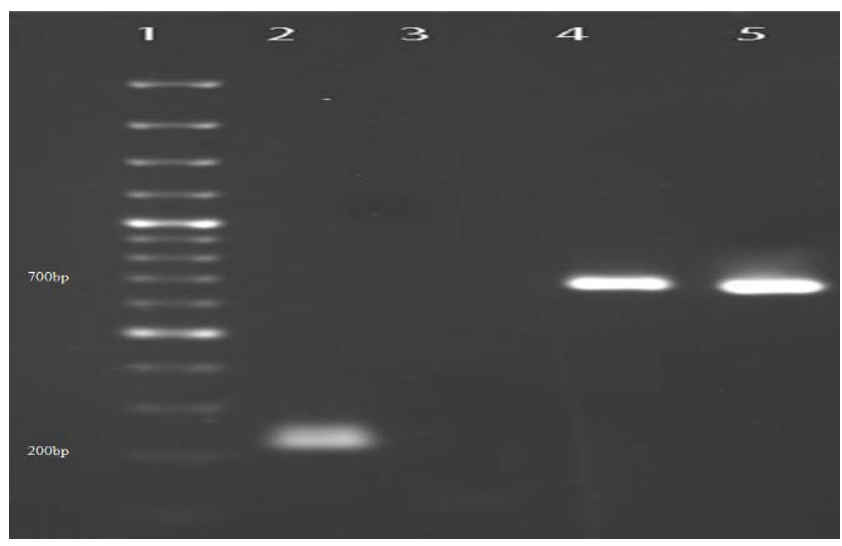

Figure 1. Semiquantitative RT-PCR of Wcor14 mRNA from acclimated plant (lane 2) and control plant (lane 3) and $\beta$-tubulin as the reference gene (lanes 4,5). Lane 1 is $100 \mathrm{bp}$ molecular weight marker

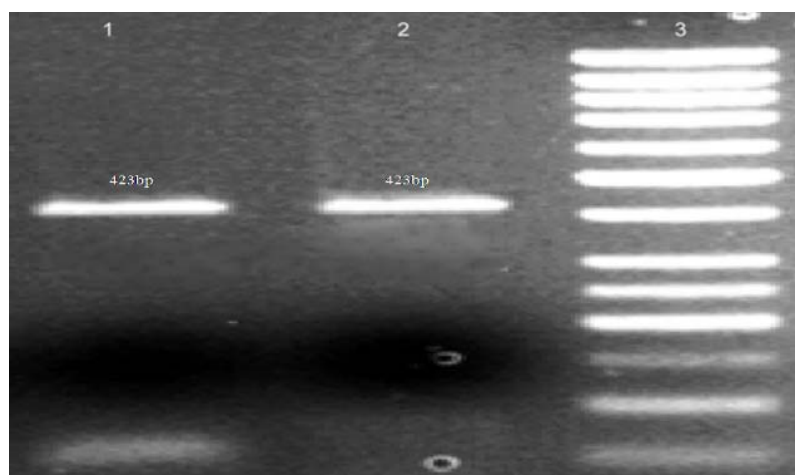

Figure 2. Electrophoresis of Wcor14 PCR products. Lane 1: Triticum aestivum, Lane 2: Ae. tauschii and Lane 3 is $50 \mathrm{bp}$ molecular weight marker

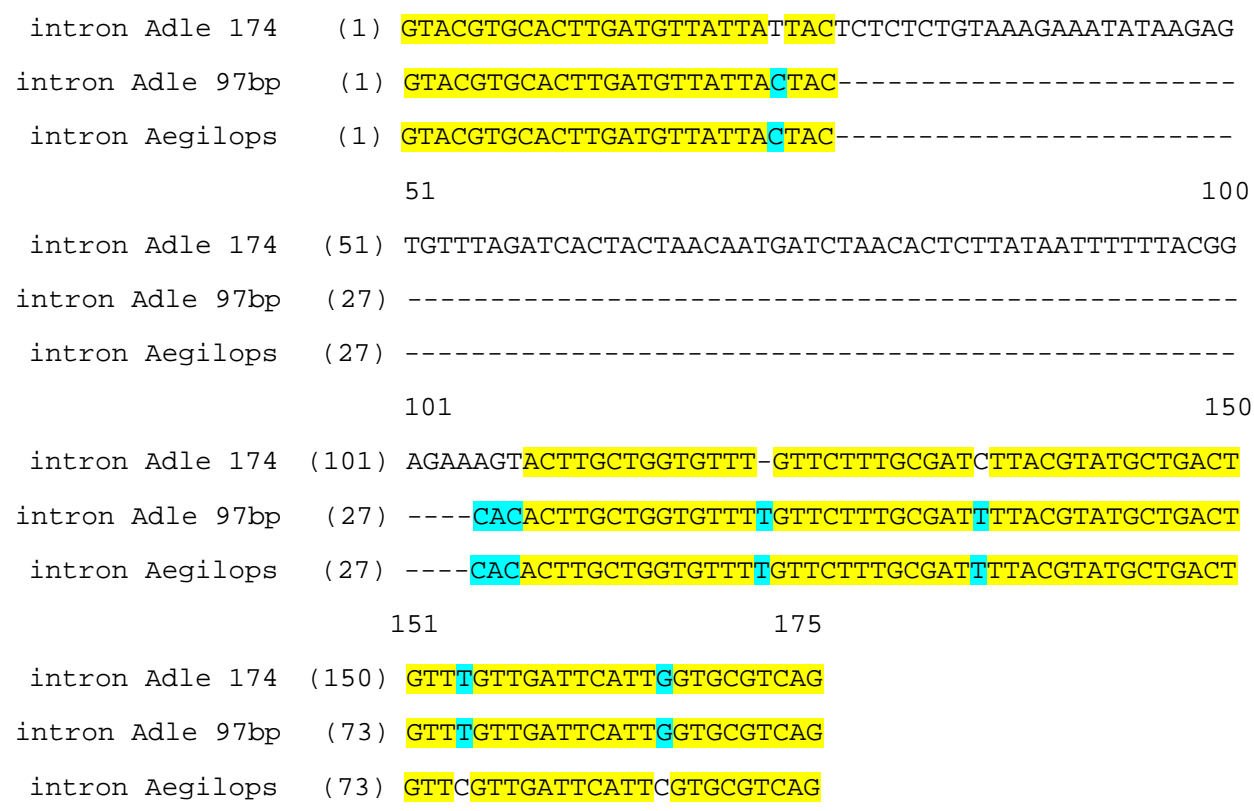

Figure 3. Alignment of intron sequences of Wcor14 loci. Introns were amplified by the nested PCR using total DNA from Triticum aestivum L. and Ae. tauschii as templates. Sequences of two different sized introns (174 bp and $97 \mathrm{bp}$ ) were detected 
A

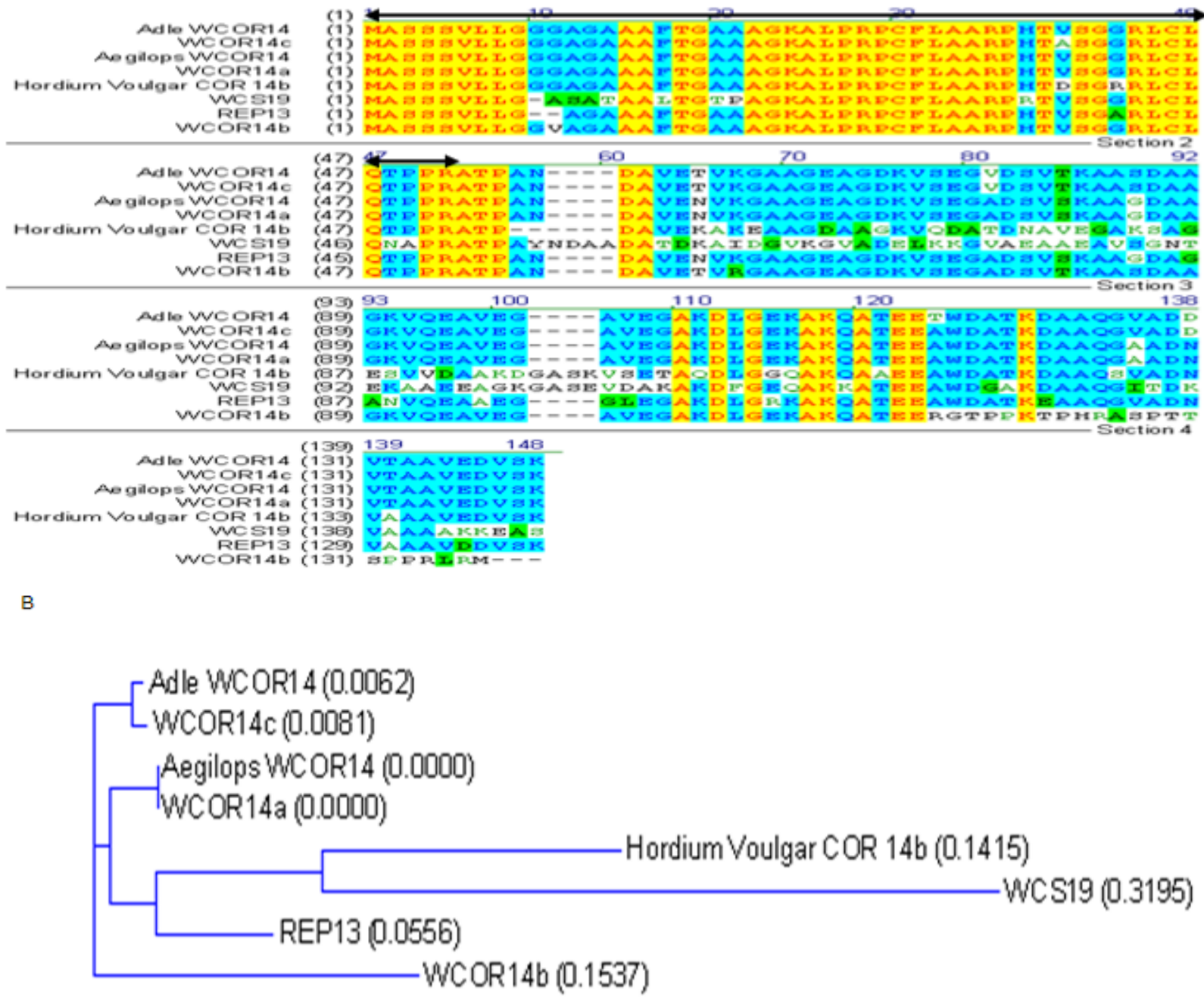

Figure 4. (A) An alignment of the deduced WCOR14 polypeptide and its homologous proteins. The N-terminal amino acid residues shown with a bold line indicate putative chloroplast signal peptides.(B) Phylogenetic tree based on amino acid sequences showing the relationship of A.tauschii COR14 and Triticum aestivum cultivar Adle cross cold-responsive protein WCOR14 with other homologous. 
MASSSVLLGGGAGAAAFTGAAAGKALPRPCFLAARPHTVSGGRLCLQTPPRATPANDAVENVKGAGGEAGDKVSEGADSV 60

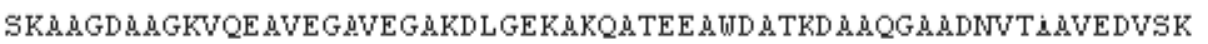

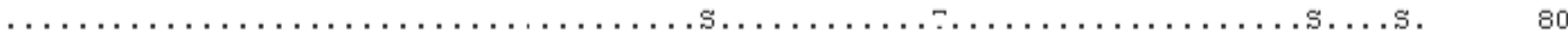

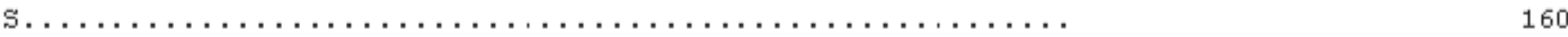

Phosphorylation sites predicted: Ser: 4 Thr: 1 Tyr: 0

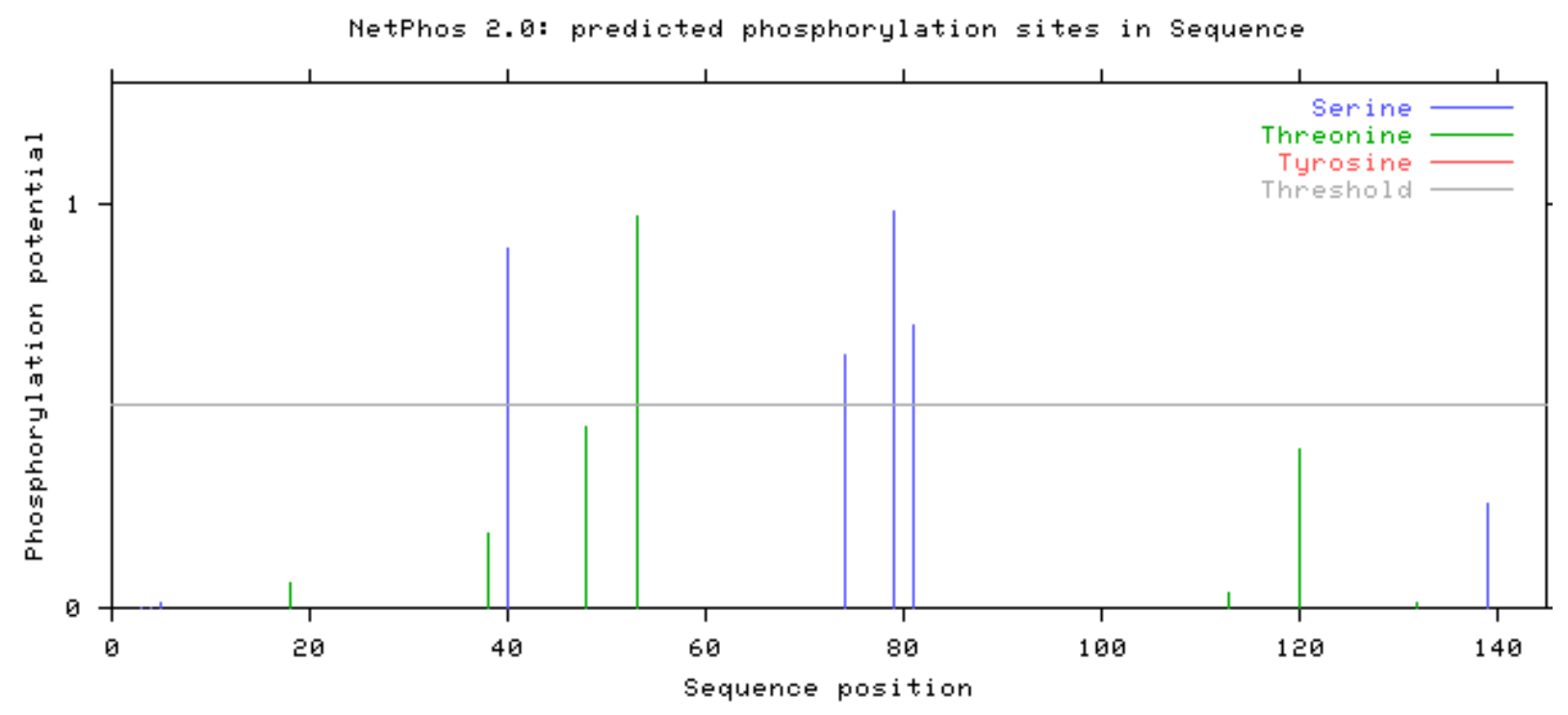

Figure 5. Predicted phosphorylation site in WCOR14 sequence.

Box show a putative 14-3-3 protein recognition motif. 
A

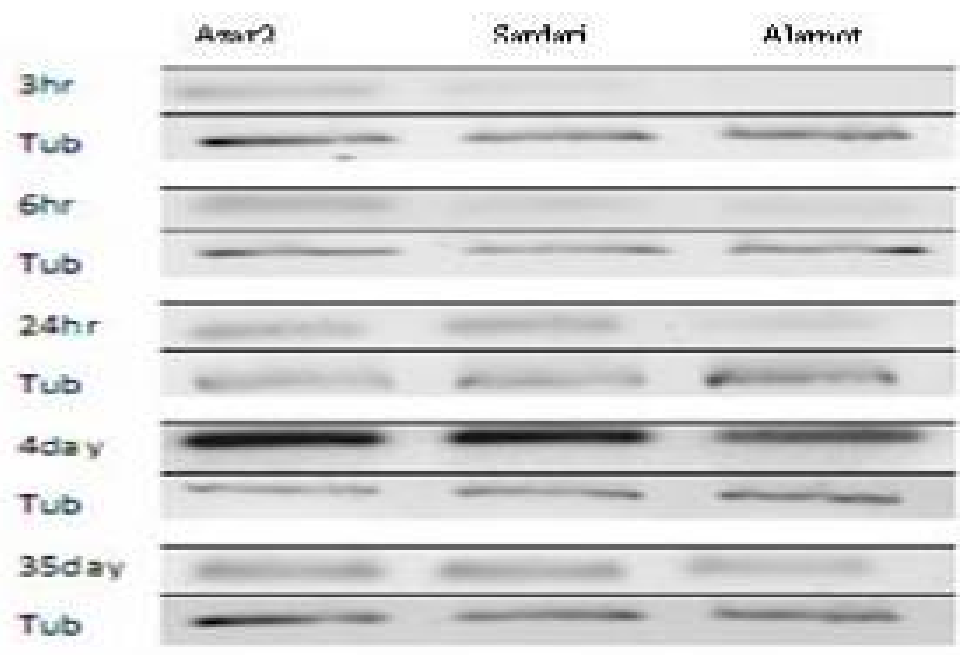

B

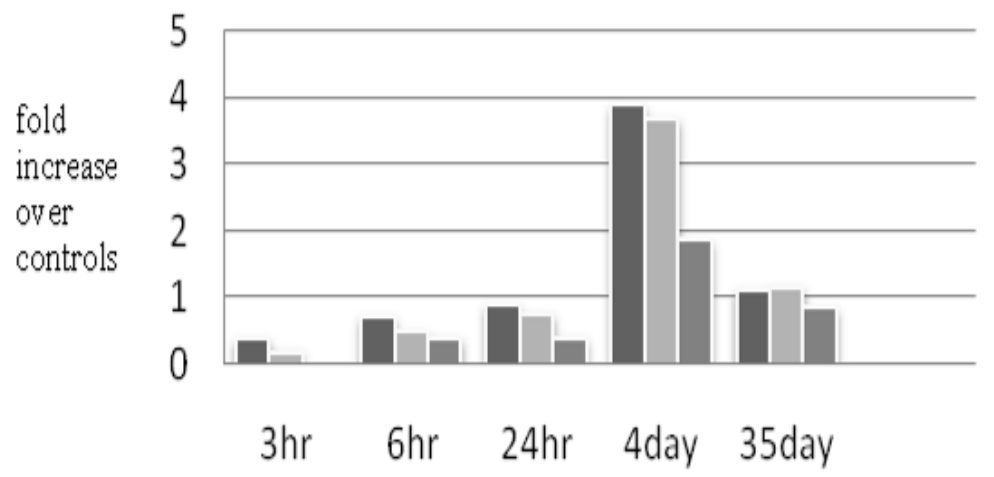

azar2 sardari alamot

Figure 6. (A) Transient enhancement of the Wcor 14 gene expression by cold stress. Total RNA was isolated from seedling leaves of Azar2, Alamoot and Sardari at the indicated time points. Semi-quantitative RT-PCR analysis was conducted to detect the Wcor 14 gene transcript. The $\beta$-tubulin gene was used as a control. (B) Quantification of the Wcorl4 transcripts in different time. Using the internal standard $\beta$-tubulin RT-PCR signal as the denominator as described in the materials and methods section. 


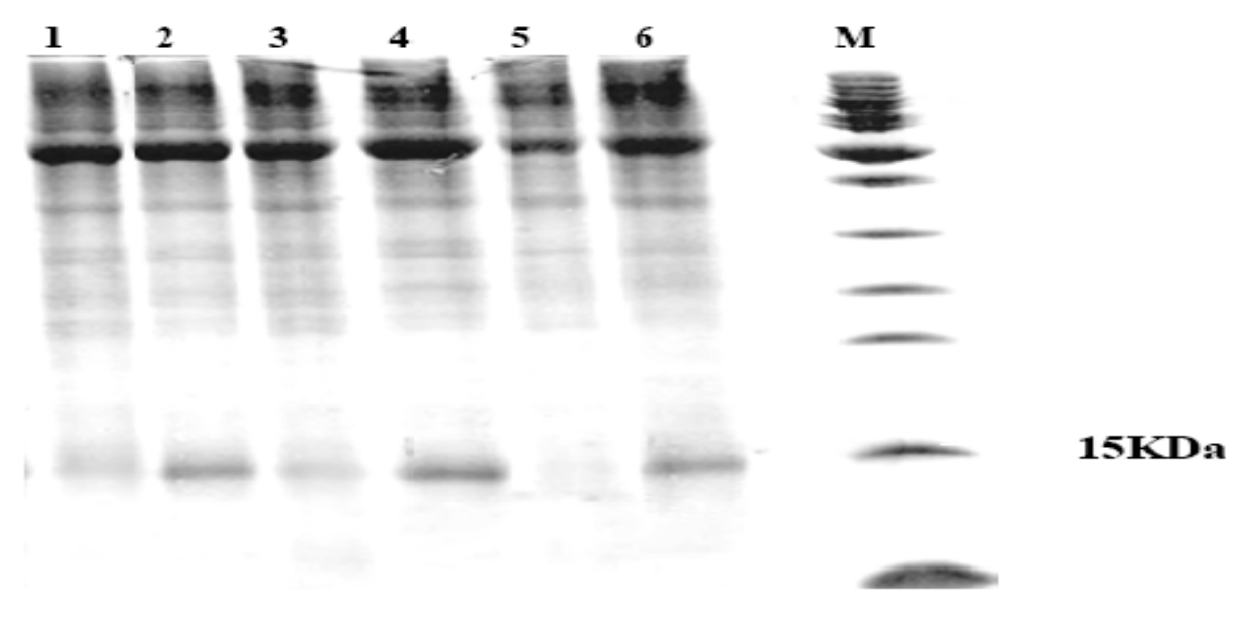

Figure 7. Electrophoresis profiles of soluble proteins in three genotypes (Azar2, Alamoot and Sardari). Lanes 1, 3, 5 profiles of Azare2, Alamoot and Sardari non-acclimated control plant, respectively. Lanes 2, 4, 6 profiles of Azare2, Alamoot and Sardari 4days cold-acclimated plant, respectively

A
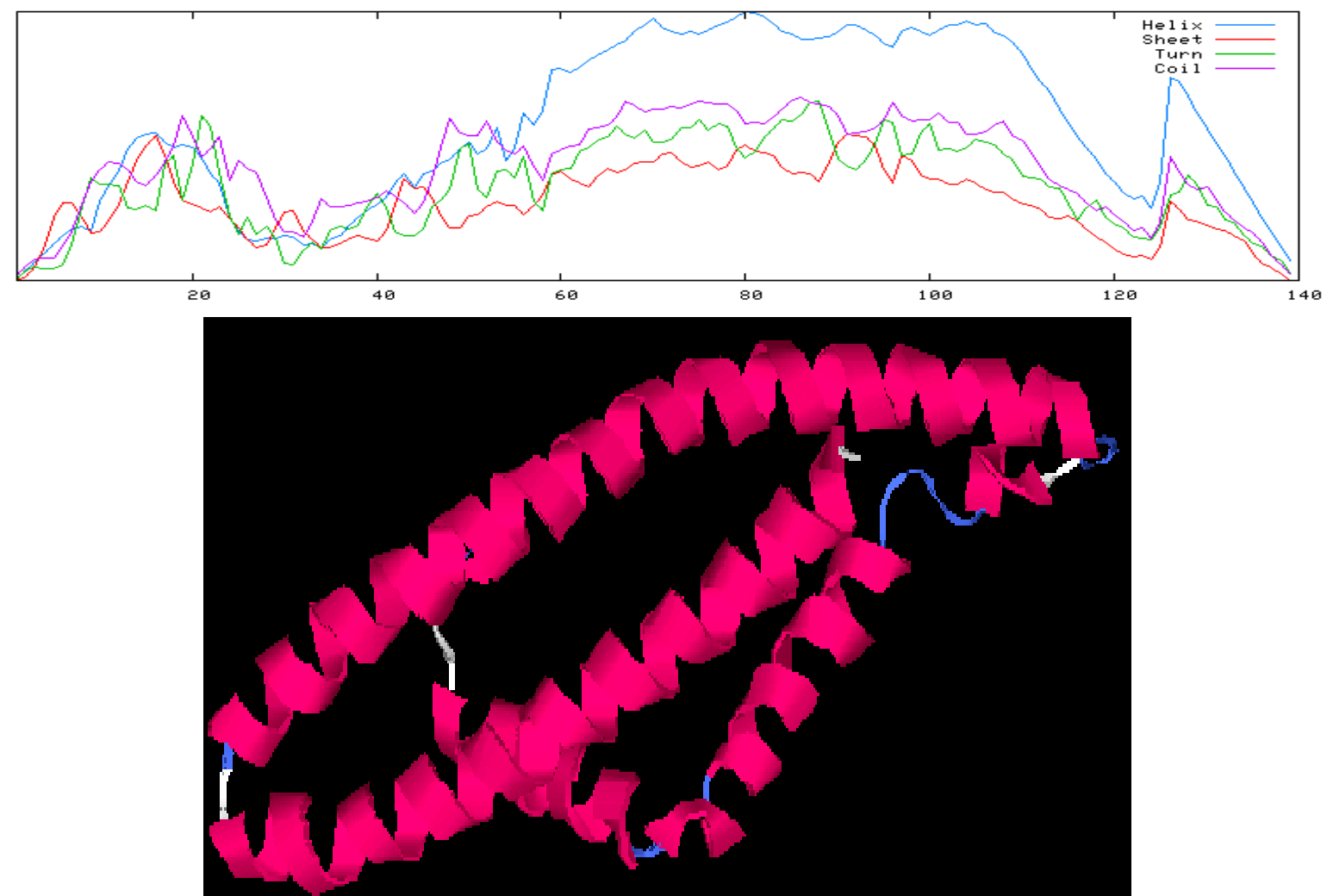
B
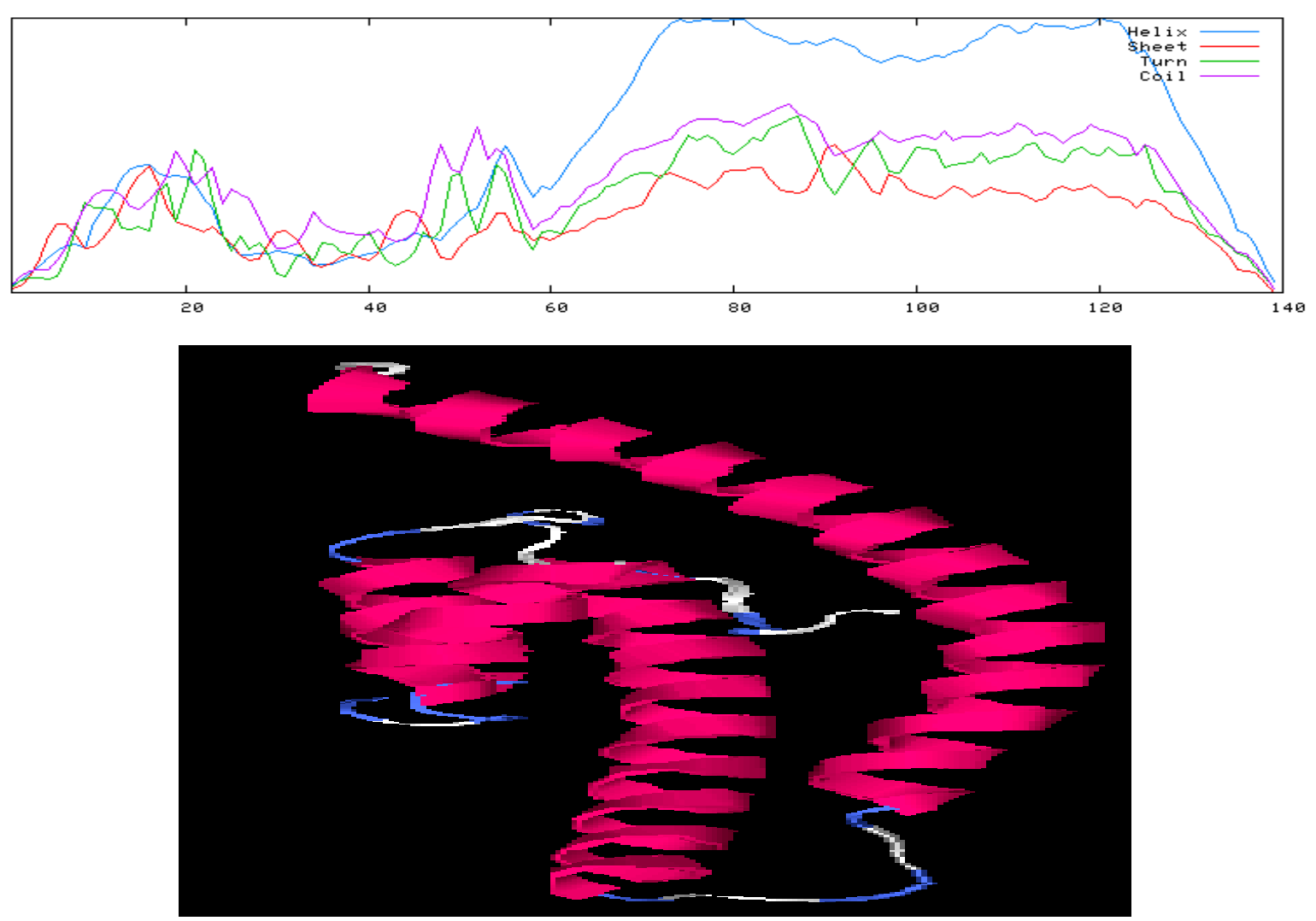

Figure 8. Predicted secondary and tertiary structure of Triticum aestivum cultivar Adle cross WCOR14 protein (A) and Aegilops tauschii WCOR14 protein (B) 